\title{
A Dynamical Principle For 3D-4D Interlinkage In Salpeter-like Equations
}

\author{
A.N.Mitra * and B.M.Sodermark* \\ 244 Tagore Park, Delhi-110009, India \\ *High Energy Lab, Dept of Phys, U of Delhi, Delhi-7 India
}

28 February 2001

\begin{abstract}
The half-century old Markov-Yukawa Transversality Principle (MYTP) which provides a theoretical rationale for the covariant instantaneous approximation $(C I A)$ that underlies all Salpeter-like equations, is generalized to a covariant null-plane ansatz $(C N P A)$. A common characteristic of both formulations is an exact 3D-4D interlinkage of BS amplitudes which facilitates a two-tier description: the $3 \mathrm{D}$ form for spectroscopy, and the 4D form for transition amplitudes as 4D loop integrals. Some basic applications of $M Y T P$ on the covariant null plane (quark mass function, vacuum condensates, and decay constants) are given on the lines of earlier applications to these processes under $C I A$.

PACS: 03.65.-w ; 03.65.Co ; 11.10.Qr ; 11.10.St

Keywords: Markov-Yukawa Transversality Principle ( $M Y T P)$; Salpeter-like eqs; Cov Instantaneity Ansatz $(C I A)$; Cov Null-Plane Ansatz (CNPA); 3D-4D interlinkage; Vertex function; 4D loops
\end{abstract}

\section{Introduction}

For a relativistic 2-body problem, the historical issue of 3D reduction from a $4 \mathrm{D}$ BSE has been in the forefront of its physics from the outset: Instantaneous approximation [1]; Quasi-potential approach [2]; variants of on-shellness of propagators [3]. This is a sort of recognition of the intractability of the strong interaction problem which had led Bethe as early as in the Fifties to invoke his famous Second Principle Theory, signifying the postulation of an effective $N-N$ interaction for a microscopic understanding of the physics of nuclei. The Bethe-Salpeter Equation (BSE) is a relativistic version of this Principle, initially at the nucleon-nucleon level, later adapted to the quark level. Now one might ask: why 3D reduction at all ? One possible answer is the need to preserve the probability interpretation which is unavailing in its $4 \mathrm{D}$ form since the BSE is only an approximate description (in the 'ladder approximation') which stems from an effective 4-fermion Lagrangian mediated by, say, a gluonic propagator which serves as the kernel of the BSE in the lowest order [4]. This may be contrasted with the Schwinger-Dyson

*e.mail: ganmitra@nde.vsnl.net.in 
Equations (SDE) which are an infinite chain of equations connecting successively higher order vertex functions [5] that stem from an exact Lagrangian which characterizes QED or QCD.

The usual 3D reduction methods [1-3] have one feature in common: The starting BSE is $4 \mathrm{D}$ in all details, including its kernel, but the associated propagators are manipulated in various ways to reduce the $4 \mathrm{D}$ BSE to a $3 \mathrm{D}$ form as a fresh starting point, giving up its original 4D form. An alternative approach which was pioneered by Weinberg [6], is intrinsically 3D in character (analogous to the Tamm-Dancoff method [7]). It was refined, among others, by Kadychevsky [8a] and Karmanov in a covariant light-front style [8b], and reviewed by the Grenoble group [9].

An alternative approach to $3 \mathrm{D}$ reduction of more recent origin $[10,11]$ is based on the Markov-Yukawa Transversality Principle (MYTP) [12], with a Lorentz-covariant 3D support postulated at the outset for the pairwise BSE kernel $K$ by demanding that it be a function of only $\hat{q}_{\mu}=q_{\mu}-q . P P_{\mu} / P^{2}$, so that $\hat{q} . P \equiv 0$; but the propagators are left untouched in their original $4 \mathrm{D}$ forms. Now unlike the traditional methods [1-3] which give only a one-way connection, $(4 D \rightarrow 3 D)$, MYTP [12] allows a two-way interconnection [11] between the 4D and 3D BSE forms, the latter rooted in spectroscopy [13].

\subsection{MYTP and 3D-4D Interlinkage of BS Amplitudes}

Our main concern in this paper is with this 'alternative approach' epitomised by the MYTP [12] which provides a rationale for the Instantaneous Approximation insofar as the latter also amounts to a 3D support to the BS kernel [10]. By the same logic, the original Salpeter Equation [1] which stems from the adiabatic approximation to the BSE [14], is also amenable to $M Y T P[12,15]$, except for the apparent loss of covariance which is a mere technicality [10]. We shall find it convenient in this paper to speak of all such BSE's with 3D support to their respective kernels as 'Salpeter-like' equations, whether at the atomic level [16] or at the quark-hadron level [17].

Since MYTP [12] is characterized by the field dependence on both coordinate and momentum, it violates local micro-causality (a basic requisite for the theory of elementary particles). QCD [18] changed this perspective by pushing the status of hadrons from the elementary to a composite level, characterized by bilocal fields [19]. Within such a bilocal scenario, the total 4-momentum $P_{\mu}$ of the composite hadron provides a naturally preferred direction which forms the basis for a covariant 3D support to the interaction kernel [1011]. For a bilocal field $\mathcal{M}(z, X)$ [19], the Transversality condition on the BS kernel was shown [20] to be equivalent to a 'gauge principle' which expresses the redundance of the longitudinal component of the relative momentum for the physical interaction between the two constituents. This in turn suffices [10] to show that the 3D Salpeter equation [1] is an exact consequence of the covariant 3D support to the Bethe-Salpeter kernel, with $P_{\mu}$ as the preferred direction, thereby giving a formal basis, not only to the 3D Salpeter equation, but also to its reconstructed $4 \mathrm{D}$ form [11]. The same logic of course goes through for the spinor case too [15].

Now the reconstructability of 4D BS amplitudes in terms of 3D ingredients had been noticed empirically [21] within the instantaneous approximation to a QCD motivated BSE framework [22], and applied to spectroscopy and processes [23]. Subsequently the Bonn Group [24], studying Salpeter-like equations with t'Hooft instantons [25], also noticed the same property. The more important issue, apparently not addressed in these approaches 
$[21,24]$, was one of support from a deeper underlying theory. This is now provided by MYTP [12], with a gauge covariant meaning [20] to the Instantaneous Approximation $(C I A)$ that characterizes all Salpeter-like equations. The 3D-4D interlinkage of BetheSalpeter amplitudes was recently generalized to the $q q q$ problem [26].

\section{Physical Ingredients for $M Y T P$ Based BSE}

The MYTP-governed BSE of course needs supplementing by physical ingredients to define a BSE kernel, much as a Hamiltonian needs a properly defined 'potential'. However its canvas is broad enough to accommodate a wide variety of kernels which must in turn be governed by independent physical principles. In this respect, the orthodox view (which we adopt) is to keep close to the traditional 4D BSE-cum-SDE methods [27] which is a spacetime extended version of NJL's [28] Dynamical Breaking of Chiral Symmetry $(D B \chi S)$ for 4-quark interaction via vector exchange [27]. This generates a mass-function $m(p)$ via Schwinger-Dyson equation (SDE) [5], which accounts for the bulk of the constituent mass of ud quarks via Politzer additivity [29]. Indeed, the BSE-SDE formalism [27] can be simply adapted [30] to the MYTP form [4] which gives 3D spectra of both hadron types [31] under a common parametrization for the gluon propagator, as well as a self-consistent SDE determination [30] of the constituent mass.

\section{2 $M Y T P$ via Covariant Null-Plane Ansatz $(C N P A)$}

Despite these attractions, the $C I A$ formulation of $M Y T P$ gives rise to ill-defined 4D loop integrals due to a 'Lorentz-mismatch' among the rest-frames of the participating hadronic composites, resulting in time-like momentum components in the (gaussian) factors associated with their vertex functions. This is especially so for triangle loops and above, such as the pion form factor and $\rho-\pi \pi$ coupling where this disease shows up as unwarranted "complexities" [32] in the amplitudes, while one- and two-quark loops [33] just escape this pathology. A possible remedy, without giving up the obvious advantage of $M Y T P$ for a 3D-4D interconnection, is a light-front/ (null-plane) formulation a la Dirac [34] by virtue of its bigger (7) stability group compared with 6 for the instant form $(C I A)$ theory. The Dirac-Weinberg theory developed into a covariant LF dynamics [35,9, 36] from a noncovariant formulation $[37,38]$ in null-plane variables. To make this language accessible to $M Y T P$ it is only necessary to generalize it from $C I A$ to a covariantly defined null-plane ansatz $(C N P A)$ which has the potential to cure the 'Lorentz mismatch' [32] disease. A recent calculation of the pion form factor, using quark triangle loops [39] suggested that this is indeed possible, so that it makes sense to systematise the CNPA formulation on closely parallel lines to $C I A[30,33]$, through a prior calibration to some standard physical processes.

\subsection{Objective and Scope of the Paper}

In this paper, we seek to generalize $M Y T P$ as an ansatz on the covariant null plane $(C N P A)$, for a treatment of Salpeter-like equations. To bring out its close similarity with the (earlier) $C I A$ formulation, we provide a summary background in Appendix A, recalling the gauge basis [20] of $M Y T P$ [12], and recapitulating the main steps under $C I A$ for an exact 3D-4D interconnection between the corresponding BS amplitudes for 
spinless quarks. Appendix B gives a corresponding derivation for the (fermionic) Salpeter equation $[10,24,15]$.

With this background, we formulate $M Y T P$ [12] on a covariant null-plane in Section 2 by demanding the BSE kernel $K$ for pairwise interaction to be a function of relative momentum $\hat{q}$ which is transverse to the composite 4-momentum $P_{\mu}$, on the lines of $C I A$, but now the third component of $\hat{q}$ must be suitably defined [39] on the Covariant null-plane as to be independent of the time-like components of $q$ at all the hadron-quark vertices of any loop. This in general makes $\hat{q}$ dependent on the orientation $n_{\mu}$ of the null-plane but this turns out to be merely a technical formality. The 3D-4D interconnection of BS amplitudes follows in close analogy to the $C I A$ case [11].

Sect.3 describes the realistic case of fermion quarks, in which the $C N P A$ formulation is made with a Gordon reduced modification of the BSE [22], again in close analogy to $C I A$. The evaluation is greatly simplified by the observation that the reduced $3 \mathrm{D} \mathrm{BSE}$ under $C N P A$ is algebraically equivalent to the corresponding $C I A$ form [30], so that the $C N P A$ extension works with the same parametrization as $C I A$ [30] which is attuned to spectroscopy [31]. However the reconstructed $4 \mathrm{D}$ vertex function in $C N P A$ is different from $C I A$. The techniques of $C N P A$ are illustrated with the typical example of $\pi \rightarrow 2 \gamma$, with normalizations sketched in Appendix $\mathrm{C}$ for both types $(P, V)$ of $q \bar{q}$ hadrons. Sections 4 and 5 give a $C N P A$ derivation for two key physical parameters i) quark mass function and ii) $q \bar{q}$ vacuum condensates, on the lines of $C I A$ [30],together with a brief comparison with perturbative QCD. Further, in view of the fundamental nature of the electroweak decay constants $f_{P}$ and $g_{V}$, Appendix D collects a quick $C N P A$ derivation of these quantities for completeness. Sect.6 concludes with a resume and a critical comparison of Salpeter-like equations with the more conventional (4D) BSE-SDE types.

\section{Salpeter-like Equations on Covariant Null Plane}

Since the central theme of the paper concerns a generalization of $M Y T P$ on the Covariant null-plane (light-front), with a view to expand its applicational base,we start by defining a 3D support to the BS kernel on the light front/null plane, on the lines of $C I A$. Now a covariant null-plane orientation may be represented by the 4 -vector $n_{\mu}$, as well as its dual $\tilde{n}_{\mu}$, obeying the normalizations $n^{2}=\tilde{n}^{2}=0$ and $n . \tilde{n}=1$. In the standard null-plane (euclidean) notation, these quantities are $n=(001 ;-i) / \sqrt{2}$ and $\tilde{n}=(001 ; i) / \sqrt{2}$, while the two transverse directions are denoted by the subscript $\perp$ on the concerned momenta. The $n$-dependence of various momenta ensures explicit covariance, whose notation is normalized to the standard null-plane notation $p_{ \pm}=p_{0} \pm p_{3}$, as $p_{+}=n . p \sqrt{2} ; p_{-}=$ $-\tilde{n} . p \sqrt{2}$, while the perp-components continue to be denoted by $p_{\perp}$ in both notations.

For the various quantities (masses, momenta, etc) we stick to the notation of [11], (see Appendix A), except when new features arise. For the relative momentum $q=$ $\hat{m}_{2} p_{1}-\hat{m}_{1} p_{2}$, where $P=p_{1}+p_{2}$ is the total 4-momentum of the hadron, the component playing the null-plane analogue of $P . q P / P^{2}$ in the instant form [11], now needs to be more carefully defined so that the time-like component does not implicitly appear; for it is this 'third component' that causes the 'Lorentz mismatch' disease by bringing in time-like components via Lorentz transformations among different vertex functions. With a little trial and error, the desired quantity turns out to be [39]

$$
q_{3 \mu}=x P_{n} n_{\mu} ; \quad P_{n}=P . \tilde{n} ; \quad x=n . q / n . P
$$


, giving $\hat{q}^{2}=q_{\perp}^{2}+z^{2} M^{2}$, as a check. We now collect the following definitions/results which will be freely used in this paper:

$$
\begin{aligned}
q_{\perp} & =q-q_{n} n ; \quad \hat{q}=q_{\perp}+x P_{n} n ; \quad x=q . n / P . n ; \quad P^{2}=-M^{2} ; \\
q_{n}, P_{n} & =\tilde{n} .(q, P) ; \hat{q} \cdot n=q . n ; \quad \hat{q} . \tilde{n}=0 ; \quad P_{\perp} \cdot q_{\perp}=0 ; \\
P . q & =P_{n} q . n+P . n q_{n} ; \quad P . \hat{q}=P_{n} q . n ; \quad \hat{q}^{2}=q_{\perp}^{2}+M^{2} x^{2}
\end{aligned}
$$

To fix the ideas, we first consider the case of spinless quarks, to be followed by the more realistic case of fermion quarks in Section 3.

\subsection{D-4D BSE on Cov. Null Plane : Spinless Quarks}

Our first task is to derive the reduced 3D BSE (wave-function $\phi$ ) from the 4D BSE with spinless quarks (wave-function $\Phi$ ) when its kernel $K$ is decreed to be independent of the component $q_{n}$, i.e., $K=K\left(\hat{q}, \hat{q}^{\prime}\right)$, with $\hat{q}=\left(q_{\perp}, x P_{n} n\right)$ [39], in accordance with the MYTP [12] condition imposed on the light front. The 4D BSE with such a kernel is, c.f., eq.(A.2):

$$
i(2 \pi)^{4} \Phi(q)=\Delta_{1}{ }^{-1} \Delta_{2}{ }^{-1} \int d^{4} q^{\prime} K\left(\hat{q}, \hat{q}^{\prime}\right) \Phi\left(q^{\prime}\right)
$$

where $m_{i}$ is the mass of quark $\# i$,

$$
\Delta_{i}=p_{i}{ }^{2}+m_{i}{ }^{2} ; \quad P^{2}=-M^{2}
$$

and $d^{4} q=d^{2} q_{\perp} d q_{3} d q_{n}$. Now define a $3 \mathrm{D}$ wave function

$$
\phi(\hat{q})=\int d q_{n} \Phi(q)
$$

and use this result on the RHS of (2.2) to give

$$
i(2 \pi)^{4} \Phi(q)=\Delta_{1}{ }^{-1} \Delta_{2}{ }^{-1} \int d^{3} q^{\prime} K\left(\hat{q}, \hat{q}^{\prime}\right) \phi\left(\hat{q}^{\prime}\right)
$$

Now integrate both sides of eq.(2.3) w.r.t. $d q_{n}$ to give a $3 \mathrm{D}$ BSE in the variable $\hat{q}_{\mu}$ :

$$
(2 \pi)^{3} D_{n}(\hat{q}) \phi(\hat{q})=\int d^{2} q_{\perp}^{\prime} d q_{3}^{\prime} K\left(\hat{q}, \hat{q}^{\prime}\right) \phi\left(\hat{q}^{\prime}\right)
$$

where the function $D_{n}(\hat{q})$, is defined as in (A.7) for $C I A[11]$ :

$$
\int d q_{n} \Delta_{1}^{-1} \Delta_{2}^{-1}=2 \pi i D_{n}^{-1}(\hat{q})
$$

and may be obtained by standard null-plane techniques $[37,23]$ as follows. In the $q_{n}$ plane, the poles of $\Delta_{1,2}$ lie on opposite sides of the real axis, so that only one pole will contribute at a time. Taking the $\Delta_{2}$-pole, which gives

$$
2 q_{n}=-\sqrt{2} q_{-}=\left[m_{2}^{2}+\left(q_{\perp}-\hat{m}_{2} P\right)^{2}\right] /\left(\hat{m}_{2} P . n-q . n\right)
$$

the residue of $\Delta_{1}$ works out from (2.1) as $2 P . q=2 P . n q_{n}+2 P_{n} q . n$, where a 'collinearity frame' $P_{\perp} . q_{\perp}=0$ [39] has been (temporarily) employed to simplify the calculations. And when the value (2.6) of $q_{n}$ is put in (2.5), one obtains (with $P_{n} P . n=-M^{2} / 2$ ):

$$
D_{n}(\hat{q})=2 P . n\left(\hat{q}^{2}-\frac{\lambda\left(M^{2}, m_{1}^{2}, m_{2}^{2}\right)}{4 M^{2}}\right) ; \quad \hat{q}^{2}=q_{\perp}^{2}+M^{2} x^{2} ; \quad x=q . n / P . n
$$


Now a comparison of (2.2) with (2.4) relates the 4D and 3D wave-fns:

$$
2 \pi i \Phi(q, P)=D_{n}(\hat{q}) \Delta_{1}{ }^{-1} \Delta_{2}{ }^{-1} \phi(\hat{q})
$$

which is valid near the bound state pole. The BS vertex function now becomes $\Gamma=$ $D_{n} \times \phi /(2 \pi i)$, just as in eq.(A.9) for $C I A$. This result is formally covariant, albeit $n_{\mu^{-}}$ dependent, yet agrees with the (apparently non-covariant) null-plane result [23] for $D_{+}$.

\section{Fermion Quarks: Full BSE Structure}

We now come to the more realistic case of fermion quarks within the SDE-BSE framework born out of an effective gluon-exchange mediated 4-fermion coupling at the input Lagrangian level with 'current' (almost massless) quarks. The gluonic propagator encompasses both the perturbative and non-perturbative regimes, and automatically preserves the chiral character of the input Lagrangian [27,30] which is broken a la $D B \chi S[28]$ in the solution of the corresponding SDE [27], albeit with MYTP constraints [4,30]. This step generates the dynamical mass function $m(p)[27,30]$ whose low momentum limit $m(0)$ gives the bulk contribution to the constituent mass $m_{\text {cons }}$, while the current mass $m_{\text {curr }}$ for $u d s$ quarks (that enter the input Lagrangian) gives a small effect. This last is in keeping with Politzer's Additivity principle [29], viz., $m_{\text {cons }}=m_{\text {curr }}+m(0)$, which provides a rationale for the quark masses usually employed in potential models. The only extra ingredient to be incorporated in this formalism is the $M Y T P$ constraint on the effective 4 -fermion interaction to have a covariant 3D support $[4,30]$. The appropriate gluon propagator between two fermion quarks which meets this requirement, must be taken in a covariant fashion [30].

Next we write down the $M Y T P$ governed BSE structure for fermion quarks with $C I A$-like support to its kernel [30], but now under $C N P A$ :

$$
i(2 \pi)^{4} \Psi(q, P)=S_{F 1}\left(p_{1}\right) S_{F 2}\left(p_{2}\right) \int d^{4} q^{\prime} K\left(\hat{q}, \hat{q}^{\prime}\right) \Psi\left(q^{\prime}, P\right) ; \quad K=F_{12} i \gamma_{\mu}^{(1)} i \gamma_{\mu}^{(2)} V\left(\hat{q}, \hat{q}^{\prime}\right)
$$

where $F_{12}$ is the color factor $\lambda_{1} \cdot \lambda_{2} / 4$ and the $V$ - function expresses the scalar structure of the gluon propagator in the perturbative (o.g.e.) plus non-perturbative regimes. The hat notation $\hat{q}_{3}$ for $C N P A$ implies that the longitudinal component is now $q_{3 \mu}=x P_{n} n_{\mu}$, with $P_{n}=P . \tilde{n}$. (Note that in a $C I A$ formulation [11,30], the corresponding longitudinal component cannot be defined so expicitly, as it gets 'mixed up' with the 'scalar' (timelike) component; this is the source of ill-defined gaussian integrals [32] under $C I A$ ). Now the full structure of $V$ under $M Y T P$ has considerable flexibility, since the only constraint is one of transversality of the relative momentum $q$ to the total momentum $P$. Nevertheless it is instructive to list a concrete form [23,31] as a prototype of the dynamics of inter-connection of the 4D amplitudes with the 3D spectroscopy in actual practice. [The interested reader should have no difficulty in setting up his own version of $V$ as long it conforms to the MYTP constraints]. Using the simplified notations $k$ for $q-q^{\prime}$, and $V(\hat{k})$ for the $V$ function, we have:

$$
\begin{gathered}
V(\hat{k})=4 \pi \alpha_{s} / \hat{k}^{2}+\frac{3}{4} \omega_{q \bar{q}}^{2} \int d \mathbf{r}\left[r^{2}\left(1+4 A_{0} \hat{m}_{1} \hat{m}_{2} M_{>}{ }^{2} r^{2}\right)^{-1 / 2}-C_{0} / \omega_{0}^{2}\right] e^{i \hat{k} \cdot \mathbf{r}} \\
\omega_{q \bar{q}}^{2}=4 M_{>} \hat{m}_{1} \hat{m}_{2} \omega_{0}^{2} \alpha_{s}\left(M_{>}^{2}\right) ; \quad \alpha_{s}\left(Q^{2}\right)=\frac{6 \pi}{33-2 n_{f}} \ln \left(M_{>} / \Lambda\right)^{-1}
\end{gathered}
$$




$$
\hat{m}_{1,2}=\left[1 \pm\left(m_{1}^{2}-m_{2}^{2}\right) / M^{2}\right] / 2 ; \quad M_{>}=\operatorname{Max}\left(M, m_{1}+m_{2}\right) ; \quad C_{0}=0.27 ; \quad A_{0}=0.0283
$$

And the values of the basic constants (all in $\mathrm{MeV}$ ) are [23,31]

$$
\omega_{0}=158 ; \quad m_{u d}=265 ; \quad m_{s}=415 ; \quad m_{c}=1530 ; \quad m_{b}=4900 .
$$

\section{1 "Off-Shell" Gordon Reduction}

The BSE form (3.1) is unfortunately not the most convenient for wider applications in practice, since the Dirac matrices entail several coupled integral equations. Indeed, it was noticed at an early stage of the BSE programme [22] (independently of MYTP!) that a considerable simplification is effected by expressing them in 'Gordon-reduced' form, (permissible on the mass shells, or better on the surface P.q $=0$ [23]), a step which may be regarded as a sort of 'analytic continuation' of the $\gamma$ - matrices to 'off-shell' regions (i.e., away from the surface P.q=0). Admittedly this constitutes a conscious departure from the original BSE structure (3.1), but such technical modifications are not unknown in the BSE literature [40] in the interest of greater manoeuvreability, without giving up the essentials. Such a step is not unreasonable, in view of the "effective" nature of the BS kernel. Moreover, the effect of this step can be strictly monitored, since the neglected effects may still be kept track of by treating the difference of the exact and the 'Gordon-reduced' kernels as a perturbation. On the other hand, the advantages of Gordon reduction are substantial, since it cures in a single stroke, a very troublesome disease which is known in the literature as a'continuum dissolution disease', first noted half a century ago [41], but revived in more recent times in the context of a 'Volks Theorem' [42] concerning the mixing of positive an negative states that is inherent in a relativistic dynamcs, which tends to produce an unrenormalizable wave function in an $n-\operatorname{body}$ system, where $n \geq 3$, while $n=2$ just escapes this pathology.

These arguments form the basis of the suggestion $[22,43]$ for a 'Gordon-reduced' form for the BSE (3.1), which stems in the first place from an effective 4-fermion interaction [4] at the Lagrangian level. To link up the Gordon-reduced fermion BSE structure with the 'scalar' form in Section 2, we first define an auxiliary function $\Phi(q, P)$ connected with $\Psi(q, P)$ as follows [43]:

$$
\Psi(q, P)=\left(m_{1}-i \gamma^{(1)} \cdot p_{1}\right)\left(m_{2}+i \gamma^{(2)} \cdot p_{2}\right) \Phi(q, P) ; \quad p_{1,2}=\hat{m}_{1,2} P \pm q .
$$

In terms of $\Phi$, eq.(3.1) in Gordon-reduced form reads as

$$
\Delta_{1} \Delta_{2} \Phi(q, P)=-i(2 \pi)^{-4} F_{12} \int d^{4} q^{\prime} V_{\mu}^{(1)} V_{\mu}^{(2)} V\left(\hat{q}, \hat{q}^{\prime}\right) \Phi\left(P, q^{\prime}\right)
$$

where the $V_{\mu}$-functions are given by $[22,23,44]$

$$
V_{\mu}^{(1,2)}= \pm 2 m_{1,2} \gamma_{\mu}^{(1,2)} ; \quad V_{\mu}^{(i)}=p_{i \mu}+p_{i \mu}^{\prime}+i \sigma_{\mu \nu}^{(i)}\left(p_{i \nu}-p_{i \nu}^{\prime}\right)
$$

Now to implement the Transversality Condition [12] for the entire kernel of eq.(3.7), all time-like components $\sigma, \sigma^{\prime}$ in the product $V^{(1)} \cdot V^{(2)}$ must first be replaced by their on - shell values. Substituting from (3.8) and simplifying gives [22, 44]

$$
\begin{gathered}
V^{(1)} \cdot V^{(2)}=4 \hat{m}_{1} \hat{m}_{2} P^{2}-\left(\hat{q}+\hat{q}^{\prime}\right)^{2}-2\left(\hat{m}_{1}-\hat{m}_{2}\right) P .\left(q+q^{\prime}\right)+" s p i n-T e r m s " \\
\text { "SpinTerms" }=-i\left(2 \hat{m}_{1} P+\hat{q}+\hat{q}^{\prime}\right)_{\mu} \sigma_{\mu \nu}^{(2)} \hat{k}_{\nu}+i\left(2 \hat{m}_{2} P-\hat{q}-\hat{q}^{\prime}\right)_{\mu} \sigma_{\mu \nu}^{(1)} \hat{k}_{\nu}+\sigma_{\lambda \mu}^{(1)} \sigma_{\lambda \nu}^{(2)} k_{\mu} k_{\nu}
\end{gathered}
$$




\subsection{Reconstruction of Fermion Vertex Fn}

Eq.(3.7) is the fermionic counterpart of (2.3) for scalar quarks, with the common scalar function $\Phi$ linking the two descriptions, so that the 3D reduction of (3.7) follows the steps (2.3) to (2.4) with the identification of $\phi$ as the appropriate 3D wave function. We skip the counterpart of eq.(2.4) for brevity, except to note that it is the appropriate dynamics for spectroscopy $[22,31]$ under $C N P A$, with a formally identical algebraic structure as in $C I A$, so that the spectroscopic predictions of both must be the same. These spectroscopic details [44], are not of immediate concern here except for the (gaussian) structure of $\phi$ :

$$
\phi(\hat{q})=\exp \left(-\frac{\hat{q}^{2}}{2 \beta^{2}}\right)
$$

where the quantity $\beta^{2}$ is dynamically determined in terms of the input quantities (3.3-5) as $[23,39,44]$

$$
\begin{aligned}
\beta^{4} & =2 \hat{m}_{1} \hat{m}_{2} M \omega_{q \bar{q}}^{2} / \gamma^{2} \\
\gamma^{2} & =1-\frac{2 \omega_{q \bar{q}}^{2} C_{0}}{M_{>} \omega_{0}^{2}} \\
M_{>} & =\sup \left(M, m_{1}+m_{2}\right)
\end{aligned}
$$

and is of course a Lorentz invariant quantity (independent of $n_{\mu}$ ).

With this knowledge of $\phi$, we may now reconstruct the $4 \mathrm{D}$ fermion vertex function in two stages. First the auxiliary scalar $\Phi(q, P)$ satisfying eq. (3.7), is treated as in the steps (2.3-2.8) to express it terms of the 3D quantities $\phi$ and $D_{n}$, viz.,

$$
\Delta_{1} \Delta_{2} \Phi(q, P)=D_{n}(\hat{q}) \frac{\phi(\hat{q})}{2 i \pi}
$$

Next the connection (3.6) between the the $4 \mathrm{D}$ fermionic $\Psi$ and the auxiliary $\Phi$ function yields $\Psi$ directly in terms of the $4 \mathrm{D}$ hadron quark vertex function:

$$
\Psi(P, q)=S_{F}\left(p_{1}\right) \Gamma(\hat{q}) \gamma_{D} S_{F}\left(-p_{2}\right) ; \quad \Gamma(\hat{q})=N_{H}\left[P_{n} / M\right] D_{n}(\hat{q}) \frac{\phi(\hat{q})}{2 i \pi}
$$

Here $\Gamma$ is a scalar factor carrying the bulk of the dynamical information, while $\gamma_{D}$ is a (kinematical) Dirac matrix which equals $\gamma_{5}$ for a P-meson, $i \gamma_{\mu}$ for a V-meson, $i \gamma_{\mu} \gamma_{5}$ for an A-meson, etc. $N_{H}$ represents the hadron normalization which is formally defined by [23]:

$$
2 i P_{\mu}=(2 \pi)^{4} \operatorname{Tr} \int d^{4} q\left[\bar{\Psi} i \gamma_{\mu} \Psi\left(m_{2}-i \gamma \cdot p_{2}\right) \hat{m}_{1}+\left(-\hat{m}_{2}\right) \bar{\Psi}\left(m_{1}+i \gamma \cdot p_{1}\right) \Psi i \gamma_{\mu}\right]
$$

where the $4 \mathrm{D}$ wave function $\Psi$, together with its adjoint $\bar{\Psi}$, are given by (3.14)and its adjoint equation respectively; $\hat{m}_{i}$, given by eq.(3.4), are the Wightman-Gaerding definitions [45, 23] of the momentum fractions carried by the two quarks. The normalizer $N_{H}$, eq.(3.15), is evaluated in Appendix $\mathrm{C}$ under both $C I A$ and $C N P A$ conditions. However, if it were regarded as the zero momentum limit of the e.m. form factor of a hadron (via quark triangle loop), it would cause Lorentz mismatch problems [32] under $C I A$, which is a principal reason [39] for recourse to $C N P A$. 
Figure 1:

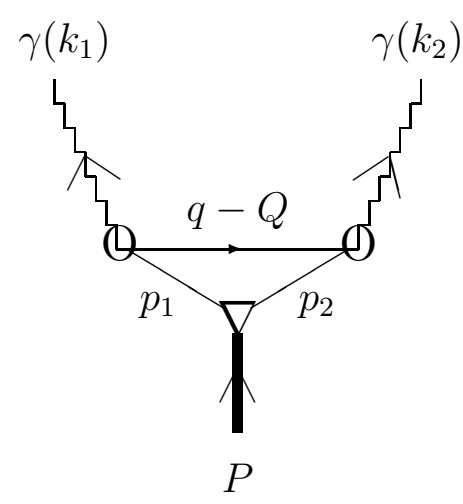

(a) $\pi^{0} \Rightarrow \gamma_{1} \gamma_{2}$

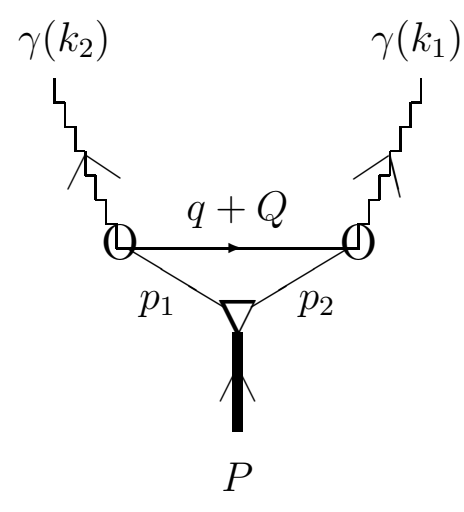

(b) $\pi^{0} \Rightarrow \gamma_{2} \gamma_{1}$

\subsection{CNPA Application to $\pi^{0} \rightarrow 2 \gamma$}

We close this Section with the example of two-photon decay of a $\pi^{0}$ meson which is given by a triangle loop. Since there is one hadron-quark vertex in this case, it does not suffer from the Lorentz mismatch problem. The invariant amplitude for $\pi^{0} \rightarrow \gamma \gamma$ decay under CNPA conditions may be written down from fig.1 below to give [11]

$$
A\left(\pi^{0} 2 \gamma\right)=\frac{1}{\sqrt{6}} e^{2} \operatorname{Tr} \int d^{4} q\left[\Psi(q, P) i \gamma \cdot \epsilon^{(1)} S_{F}(q-Q) i \gamma \cdot \epsilon^{(2)}+1 \Rightarrow 2\right]
$$

where $2 q=p_{1}-p_{2}$ and $2 Q=k_{1}-k_{2}$, and the color and flavour factors have been taken in the standard way. The second term corresponds to an interchange of the two photons. This general structure defines the $\pi^{0} \gamma \gamma$ form factor $F_{\pi}$ through the relation [46]

$$
A\left(\pi^{0} 2 \gamma\right) \equiv F_{\pi} \epsilon_{\mu \nu \rho \sigma} \epsilon_{\mu}^{(1)} \epsilon_{\nu}^{(2)} P_{\rho} Q_{\sigma}
$$

Evaluating the traces in (3.16) after substitution from (3.14), and a routine simplification, leads to the identification

$$
F_{\pi}=\frac{e^{2}}{\sqrt{6(2 \pi)^{3}}} \frac{4 m_{q} N_{\pi}}{2 i \pi} \int d^{4} q \frac{D_{n} \phi}{\Delta_{1} \Delta_{2}}\left[\frac{1}{\Delta_{3}^{+}}+\frac{1}{\Delta_{3}^{-}}\right]
$$

where

$$
\Delta_{1,2}=m_{q}^{2}+p_{1,2}^{2} ; \quad \Delta_{3}^{ \pm}=m_{q}^{2}+(q \mp Q)^{2}
$$

The gauge invariance is of course explicit from the structure of (3.17). To simplify (3.18), the Lorentz invariant measure

$$
d^{4} q=d^{2} q_{\perp} d(q . n) d\left(q_{n}\right)
$$

may be used first to integrate over $d\left(q_{n}\right)$, to yield a remarkably simple yet accurate result:

$$
\int d\left(q_{n}\right) \frac{D_{n} \phi}{\Delta_{1} \Delta_{2}}\left[\frac{1}{\Delta_{3}^{+}}+\frac{1}{\Delta_{3}^{-}}\right] \approx \frac{2 i \pi \phi}{m_{q}^{2}+q_{\perp}^{2}}
$$


which is a $C N P A$ adaptation of the corresponding result [47] on $\pi^{0} 2 \gamma$ decay in usual null-plane variables [37]. (For details of steps on null-plane pole integrations in the $p_{2-}$ variable, see ref.[47]). Eq.(3.18) then simplifies to

$$
F_{\pi}=\frac{4 e^{2} m_{q} N_{\pi}}{\sqrt{6(2 \pi)^{3}}} \int \frac{d^{2} q_{\perp} M d x \phi}{m_{q}^{2}+q_{\perp}^{2}}
$$

where $\phi$ is given by eq.(3.11), with $\hat{q}^{2}=q_{\perp}^{2}+x^{2} M^{2}$. The integral finally works out at

$$
F_{\pi}=\frac{4 e^{2} m_{q} N_{\pi} \beta^{3}}{\sqrt{6}} \operatorname{erf}\left(\sqrt{M^{2} / 8 \beta^{2}}\right) \int_{0}^{\infty} d x \frac{e^{-x}}{m_{q}^{2}+2 \beta^{2} x}
$$

The decay rate in turn is given by [46]

$$
\Gamma\left(\pi^{0} \rightarrow 2 \gamma\right)=\frac{F_{\pi}^{2} M^{3}}{64 \pi}
$$

Using the value of $N_{\pi}=31.88 \mathrm{GeV}^{-3}$ after substitution from eq.(C.5) of Appendix $\mathrm{C}, F_{\pi}$ is predicted as $29 \mathrm{MeV}^{-1}$, leading to the value $11 \mathrm{ev}$ which agrees with the $C I A$ value [11] but is about $30 \%$ higher than the observed value of 8.5ev [13]. We note in passing that an alternative formulation in terms of "half-off-shell" wave functions in null-plane variables [23] gives a much closer agreement with experiment [13]. However such wave functions [23], although conforming to the Weinberg [6] spirit of the infinite momentum frame, fail to satisfy the angular condition $[35,9]$ necessitated by $O(3)$ invariance.

\section{Dynamical Mass Via $D B \chi S$ Scenario}

The 'dynamical' mass function of the quark may be defined in one of two ways: i) as the non-trivial solution of the SDE [27] under $D B \chi S$ [28]; ii) as the vertex function $\Gamma(\hat{q})$, for the pion in the chiral limit $\left(M_{\pi}^{2}=0\right)$. The logic of the second form follows from the original NJL paper [28] for contact interaction, which was subsequently found to be more generally satisfied for extended 4-fermion interaction with vector exchange [27] whose chiral invariance ensures that the SDE for the self-energy operator $\Sigma(p)$ (essentially the quark mass function $m(p)$ ), and the BSE for the pion-quark vertex function $\Gamma(q, P)$ are formally identical in the limit of zero pion 4-momentum, leading to the conclusion that these two functions are basically the same, except for the normalization. This result is also valid for the MYTP oriented 3D-4D BSE formalism [30], except for the replacement of $m(p)$ by $m(\hat{p})$, and offers a practical way to construct the mass function in terms of the pion-quark vertex function via the BSE route for hadron-quark interaction [30].

\subsection{Mass Fn as $D B \chi S$ Limit of Pion Vertex Fn}

The general hadron-quark vertex function is proportional to the product $D(\hat{q}) \times \phi(\hat{q})$, so that the mass function $m(\hat{p})$ is obtained by setting $M_{\pi}=0$ in this expression in the limit $P_{\mu}=0$, where $p_{\mu}$ is now the 4-momentum of either quark. Making the necessary substitutions, the mass function is identified in $C I A$ as [30]:

$$
m(\hat{p})=\frac{\omega^{3}(\hat{p})}{m_{q}^{2}} \phi(\hat{p}) ; \quad \omega^{2}(\hat{q})=m_{q}^{2}+\hat{p}^{2},
$$


normalized to the 'constituent' mass $m_{q}$ in the limit of $\hat{p}=0$. As a simple check, the mass function vanishes in the $p \rightarrow \infty$ limit. The 3D wave function $\phi$ has the gaussian form (3.11), with $\beta^{2}=0.060 \mathrm{GeV}^{2}[39]$ after substitution from eq(3.12), together with (3.4-5), for the pion case.

We now turn to the corresponding derivation under $C N P A$ in close parallel to above [30], except for the definition of the denominator function which we write in the 'standard' null-plane notation [37-38,23] for easier comparison with $C I A$ :

$$
D_{n}=2 P_{+}\left(m_{q}^{2}+\hat{q}^{2}-M^{2} / 4\right) ; \quad \hat{q}^{2}=q_{\perp}^{2}+M^{2} q_{+}^{2} / P_{+}^{2}
$$

The factor in front shows that the role of $2 \omega(\hat{q})$ in the instant form $(C I A)$ is now played by $P_{+}$in the null-plane form. This is in conformity with the Dirac-Weinberg notion $[34,6]$ of the 'plus' component as the 'mass' term, which is of course orientation $\left(n_{\mu}\right)$-dependent. The $C N P A$ mass function is now

$$
m_{+}(\hat{p})=p_{+} \frac{\left(m_{q}^{2}+\hat{p}^{2}\right) \phi(\hat{p})}{m_{q}^{2}} ; \quad p_{+}=\sqrt{2} p . n
$$

in the same relative normalization as in eq.(3.1), and with the replacement $P_{+}\left(=p_{1+}+\right.$ $\left.p_{2+}\right) \Rightarrow 2 p_{+}$in the chiral limit.

This form of the mass function is convenient for applications to certain types of loop integrals such as vacuum condensates [30] among other things. It is not of course Lorentz invariant by itself, unlike in standard 4D SDE-BSE formalism [27], but this is not a serious problem since it is not a directly measurable quantity except in the limits of $p \rightarrow 0$ (constituent mass), or $p \rightarrow \infty$ (current mass), where it is Lorentz invariant. However it yields Lorentz invariant quantities where it enters as a dynamical ingredient, e.g., in the evaluation of vacuum condensates [30]; see Section 5.

\subsection{Dynamical Mass from SDE for $\Sigma(p)$}

The more standard aspect of the 'dynamical' mass function is its appearance as the nontrivial solution of the SDE under $D B \chi S$ [27]. We now give a summary derivation of the $3 \mathrm{D}-4 \mathrm{D}$ counterpart of this basic result, which although obtained under $C I A$ premises, is almost literally valid for $C N P A$, with the replacement $k_{l} \rightarrow k_{n}$. To that end we start with the non-perturbative part of the gluon propagator $D_{\mu \nu}(k)=D(k)\left[\delta_{\mu \nu}-k_{\mu} k_{\nu} / k^{2}\right]$ for the (harmonic) interaction of $u d$ quarks where the scalar factor $D(k)$ has the form [30]

$$
D(k)=\frac{3}{4}(2 \pi)^{3} \omega_{0}^{2} 2 m_{q} \alpha_{s}\left(4 m_{q}^{2}\right)\left[\nabla_{\hat{k}}^{2}+C_{0} / \omega_{0}^{2}\right] \delta^{3}(\hat{k})
$$

which is immediately derivable from the structure of the 'potential' function $V \hat{k})$, eq( $(3.2)$, with the $A_{0}$-term dropped as insignificant for this case, and taking $M_{>}=2 m_{q}$ for the 'pion'. Note that $D(\hat{k})$ has a directional dependence $n_{\mu}=P_{\mu} / \sqrt{P^{2}}$ on the pion 4momentum $P_{\mu}$, so that $\hat{k}^{2}>0$ over all $4 \mathrm{D}$ space; it also possesses a well-defined limit for $P_{\mu} \rightarrow 0$. This structure may now be substituted in the SDE for a self-consistent solution in the low momentum limit, which in the Landau gauge $A\left(p^{2}\right)=1$ [48] becomes [30]

$$
m(p)=\frac{3 i}{\pi} \int d^{3} \hat{k} d k_{0} m_{q} \alpha_{s}\left[\omega_{0}^{2} \nabla_{\hat{k}}^{2}+C_{0}\right] \delta^{3}(\hat{k}) \frac{m\left(p^{\prime 2}\right)}{\left[p^{\prime} 2+m^{2}\left(p^{\prime 2}\right)\right]}
$$


where $p^{\prime}=p-k$ is $4 \mathrm{D}$, and $\left(\hat{k}, k_{0}\right)$ are $(3 \mathrm{D}, 1 \mathrm{D})$ respectively. The integration is essentially over the time-like $k_{0}$, with the 'pole' position at $p_{0}{ }^{\prime}=m\left(p_{0}^{\prime}\right) \equiv m_{N J L}$, leading finally to $[30]$

$$
m_{N J L}=\frac{3 m_{q} \alpha_{s}}{m_{N J L}{ }^{2}}\left[3 \omega_{0}^{2}-C_{0} m_{N J L}^{2}\right] ; \quad \alpha_{s}=\frac{6 \pi / 29}{\ln \left(10 m_{q}\right)}
$$

after substituting the values (3.4-5) for the QCD constant $\Lambda$, etc. The further identification of $m_{q}$ with $m_{N J L}$ in this equation, yields an independent self-consistent estimate $m_{N J L} \sim 300 \mathrm{MeV}$, which may be compared to the input value $265 \mathrm{MeV}$, eq.(3.5) used for the spectra [31]. Thus the use of the SDE in conjunction with the BSE provides a powerful check on the consistency of the otherwise empirical constituent mass which is no longer a free parameter. This analysis so far ignores the Politzer relation [29] $m_{u d}=m_{c}+m_{N J L}$, for the constituent mass $m_{q}$ away from the chiral limit; for this extended derivation see [30].

We end this Section with some comments on the interpretation of the two basic constants $C_{0}$ and $\omega_{0}$, in view of their appearance in the determining equation (4.6) for the constituent mass $m_{N J L}$. From eq.(3.3), $\omega_{0}$ may be regarded as a 'reduced spring constant' of the confining interaction for light quarks (for which the constant $A_{0}$ is not important). It controls the confinement scale [30] for a hopefully integrated view of the different flavour sectors of hadron spectra [31]. $C_{0}$ is a second constant designed to simulate the zero-point (vacuum) energy effects via the replacement $r^{2} \rightarrow r^{2}-C_{0} / \omega_{0}^{2}$. Both these quantities are as fundamental in a 'potential' model context [17], as the pionic constant $f_{\pi}$ is in, say, chiral perturbation theory [49], or the role that vacuum condensates play as cofficients of the successive 'twist' terms in the Wilson OPE expansion employed in QCD sum rules [50]. In the present state of the QCD art, it is perhaps a matter of taste as to which set of constants should be considered as more basic than the other, but the facility of a derivation of the latter in terms of the former, as partly illustrated in the foregoing, should hopefully constitute a connection between the two languages, with the advantage of the 'spectroscopic link' associated with the former [30]. The formal possibility of a selfconsistent derivation of $m_{N J L}$ in terms of $\omega_{0}$ and $C_{0}$, as illustrated above (while leaving scope for quantitative corrections due to the neglected effects such as the oge term), is one such manifestation of this connection.

\section{Direct Calculation Of Quark Condensates}

As was first shown by the Orsay group [51], the 'potential' method offers a direct method of calculation of the condensate, in terms of the quark's non-perturbative mass function $m(p)$ as the chiral $\left(M_{\pi}=0\right)$ limit of the pion-quark vertex function $\Gamma(\hat{q})$, viz., eq. (4.1) for $C I A$ or (4.3) for $C N P A$. This function must be used in the expression for the full propagator, $S_{F}(p)$ which appears in the formal definition of the condensate as follows:

$$
<\bar{q} q>=\frac{i N_{c} N_{f}}{(2 \pi)^{4}} \operatorname{Tr}\left[\int d^{4} p S_{F}(p)\right]
$$

where

$$
S_{F}(p)=\frac{-i}{m(p)+i \gamma \cdot p}
$$


in the Landau gauge [48]. Here $N_{c}=3$, and $N_{f}=1$ (since each separate flavour $(u / d$ ) is counted). In the MYTP scenario, the mass function does not depend on the time-like component of $p_{\mu}$. Therefore after taking the traces on the RHS of (5.1), and doing the pole-integration over the time-like component of $p_{\mu}$, the above equation becomes for $C I A$

$$
<\bar{q} q>=-\frac{3}{4 \pi^{3}} \int d^{3} \hat{p} \frac{m(\hat{p})}{\sqrt{\hat{p}^{2}+m^{2}(\hat{p})}}
$$

To evaluate the 3D integral (5.2) further, substitute the $C I A$ structure (4.1) for $m(\hat{p})$, with $\phi(\hat{p})=\exp \left(-\hat{p}^{2} / 2 \beta^{2}\right)$, which gives a simple quadrature for the resulting integral. Further, since the integral has an analytic form in $m_{q}$, it is useful for evaluating a related quantity, viz., the 'increment' $\delta<\bar{q} q>$ due to a shift $\delta m_{q}$ in the 'constituent' mass, which by Politzer Additivity [29] equals a corresponding shift $\delta m_{c}$ in the 'current' mass. Both these parameters are directly comparable with corresponding estimates from QCD sum rules [50]. Using the inputs from (3.4-5) gives $\beta^{2}=0.0603$, and the final results for this case are $[52]$

$$
<\bar{q} q>=-(266 \mathrm{MeV})^{3} ; \quad \delta<\bar{q} q>=+0.0664 \delta m_{c}
$$

These values are fully rooted in spectroscopy but are otherwise free from adjustable parameters, except for the quantity $\delta m_{c}$ which represents the $u-d$ mass difference. The condensate has a fair overlap with QCD-SR determinations [48], but its increment is rather small (for possible reasons, see below).

In a similar way the corresponding condensate results under $C N P A$ are found by substituting (4.3) in (5.1), to give

$$
<\bar{q} q>=\frac{12 i \sqrt{2}}{(2 \pi)^{4}} \int d^{3} \hat{p} d p_{n} \frac{p \cdot n\left[1+\frac{\hat{p}^{2}}{m_{q}^{2}}\right] \phi(\hat{p})}{m_{q}^{2}+p_{\perp}^{2}-2 p \cdot n p_{n}}
$$

The integration over $p_{n}$ is again trivial and the $C N P A$ counterpart of (5.2) is :

$$
<\bar{q} q>=\frac{-3 \sqrt{2}}{(2 \pi)^{3}} \int d^{3} \hat{p}\left[1+\frac{\hat{p}^{2}}{m_{q}^{2}}\right] \phi(\hat{p})
$$

Substituting the gaussian form for $\phi$ and integrating, yields a simple analytic form:

$$
<\bar{q} q>=-3 \sqrt{2}\left(\beta^{2} / 2 \pi\right)^{3 / 2}\left[1+3 \beta^{2} / m_{q}^{2}\right]=-(242 M e V)^{3}
$$

a value which seems to be even closer to the estimate $-(240)^{3}$ of QCD-SR [48] than the $C I A$ result $-(266)^{3}[52]$.

We end this Section by noting that the quantity $\delta<\bar{q} q>$ offers a comparison with QCD-SR [50] in terms of its effect on certain physical quantities derivable from it. Thus it contributes to hadron mass splittings due to strong $\mathrm{SU}(2)$ breaking [52], albeit by a small amount. This contrasts with the corresponding QCD-SR findings [53] that suggest dominance of this very contribution. This is not surprising since within a BSE-cum-SDE framework, most of the non-perturbative effects are already contained in the hadron-quark vertex function, with a correspondingly smaller role for the condensates. This philosophy of the BSE-SDE formalism is somewhat akin to that of the Pagels-Stokar [54] "Dynamical Perturbation Theory" (neglect of 'criss-cross' gluon lines in a loop diagram), which must be carefully distinguished from a naive interpration of perturbative QCD. On the other hand in a QCD-SR scenario [50] such condensate contributions which arise from the 'twist terms' in an OPE expansion, are perhaps the dominant source of non-perturbative effects. 


\section{Resume And Conclusions}

Salpeter-like equations [1] which may be defined as BSE's with 3D kernel support, have the property of exact 3D reduction, as well as reconstruction of the $4 \mathrm{D}$ amplitude in terms of 3D ingredients. The fact that such equations are governed by a well-defined dynamical principle (with a gauge content [20]), known as the the Markov-Yukawa Transversality Principle $(M Y T P)[12$, gives them a unique status in the contemporary literature, characterized as they are by a two-tier dynamics, the 3D form for $O(3)$-like spectra, and the $4 \mathrm{D}$ form for transition amplitudes as 4D loop integrals. However the vehicle of $C I A$ through which MYTP has operated so far, suffers from a sort of "Lorentz incompatibility" [39] among the participating vertices in triangle loops and above. These show up as ill-defined integrals due to the presence of time-like momenta in the (gaussian) form factors, leading to complexities in amplitudes [32], while loops involving up to two quark lines [33] just escape this pathology. To deal with this problem, we have proposed a generalization from $C I A$ to a covariantly defined null-plane ansatz $(C N P A)$ which retains the property of 3D-4D interlinkage, but does not suffer from the problem of time-like

gaussians in the loop integrals, albeit at the cost of dependence on the orientation $n_{\mu}$ of the null-plane [39]. However, as found from a recent calculation of the pion form factor [39], the $n$-dependence is a mere technicality which may be trivially eliminated via the 'Lorentz completion' trick leading to an explicitly Lorentz-invariant structure [39]. In this paper an attempt has been made for a systematic development of the $C N P A$ framework on closely parallel lines to $C I A$ through a few basic calibrations (the quark mass function, quark condensate and electroweak constants). Comparison with the $C I A$ framework is further facilitated by the fact that the reduced $3 \mathrm{D}$ forms have formally identical structures for both. However the predictions differ at the level of loop integrals: The difference is small for two-quark loops, but only $C N P A$ seems to make sense for triangle (and higher) loops [39], for which $C I A$ is ill-defined.

\subsection{What Distinguishes Salpeter-like Eqs From Others ?}

We conclude with a summary of some salient features of Salpeter-like Eqs [1] which distinguish them from most other 3D approaches to strong interaction dynamics [1-3], including null-plane dynamics $[6,9,35]$.

A first comparison of Salpeter-like Eqs. with a standard 4D BSE [27, 55] concerns the role of some additional length scales in the infra-red part of the gluon propagator [55] which are not quite 'tested' without considering $L$ - excited spectra, for which there is no evidence yet [55]. MYTP-based Salpeter-like Eqs, on the other hand, allow a more explicit test of the gluon propagator [31] in respect of both ground and $L$-excited states.

Another aspect concerns the question of the 3D support ansatz going beyond the conventional ladder approximation, since the very interpretation of the quantity $m_{N J L}$ as a 'constituent mass' could otherwise be questioned on the ground that its generation requires the presence of a second source of color charge [56], while the solution of the SDE in the rainbow approximation [55] misses this detail due to the dependence of the standard oge propagator on a single 4 -vector $k_{\mu}$ only. On the other hand, a Salpeter-like Eq, via the 3D support ansatz, effectively ensures that the oge propagator "sees" [30] the second source through its directional dependence on the composite 4-momentum $P_{\mu}$, in addition to $k_{\mu}$. Indeed the identification of the mass function $m(p)$ as the chiral limit 
$\left(P_{\mu} \rightarrow 0\right)$ of the pion-quark vertex function $\Gamma(q, P)$ (which in turn is a solution of a 2-body equation), would not be consistent if $m(p)$ were to depend on $p_{\mu}$ alone. A Salpeter-like Eq, with its $M Y T P$ based dependence on

$$
\hat{p}_{\mu}=p_{\mu}-p \cdot P P_{\mu} / P^{2}
$$

makes this function logically more consistent with the concept of a second source of color charge $[30,56]$.

A last item of comparison concerns the elimination of a class of singularities which would appear in a 4D loop integral due to overlapping pole effects, viz., the LandauCutkowsky singularities which are usually responsible for 'free' propagation of quarks inside closed loops. According to the standard wisdom [57], the infrared behaviour of the gluon propagator helps tone down the effect of this pole, via WT identities, but doubts have also been expressed [57] about the uniqueness of the procedure. In an (MYTP-governed) Salpeter-like Eq, the characteristic $D \times \phi$ structure of the hadronquark vertex function automatically ensures that the $D$-function simply cancels out such Landau-Cutkowsky poles, and thus prevents the free propagation of quarks. This is true for both $C I A[11]$ and $C N P A[39]$ by virtue of the $D \times \phi$ form of the vertex function.

The 3D-4D interlinkage offered by $M Y T P$ is also generalizable to a 3-body BSE with pairwise kernels under covariant 3D support [26]. Other applications include 3-hadron couplings like $\rho-\pi-\pi, \omega-\rho-\pi$, tests of Vector Meson Dominance, etc., some of which are under way.

One of us (BMS) is grateful to Prof R.K.Shivpuri for the hospitality of the High Energy Lab at Delhi University.

\section{Appendix A. MYTP As A Gauge Principle}

In this Appendix we summarise the gauge aspects of MYTP [12b] which are brought out by the dynamics of bilocal fields [19] and demonstrate the 3D-4D interlinkage of BS amplitudes [11]. Now the gauge principle underlying MYTP asserts the redundance [20] of the relative 'time' variable $x_{0},\left(x=x_{1}-x_{2}\right)$, whose covariant definition is just the longitudinal component of $x_{\mu}$ in the direction of $P_{\mu}$, viz., $x . P P_{\mu} / P^{2}$. This 'redundance' is expressed by the statement that a translation of the relative coordinate $[20] x_{\mu} \rightarrow x_{\mu}^{\prime}+\xi P_{\mu}$ on the bilocal field $\mathcal{M}(x, P)$ :

$$
\mathcal{M}\left(x_{\mu}, P_{\mu}\right) \rightarrow \mathcal{M}_{\xi}\left(x_{\mu}, P_{\mu}\right)=\mathcal{M}\left(x_{\mu}+\xi P_{\mu}, P_{\mu}\right)
$$

, which is a sort of 'gauge transformation' for the bilocal field [20], should leave this quantity invariant. This invariance is just the content of the Markov-Yukawa subsidiary condition [12] which, under an interchange of the relative coordinates and the momenta reads as $[20,5 b]$

$$
P_{\mu} \frac{\partial}{\partial x_{\mu}} \mathcal{M}\left(x_{\mu}, P_{\mu}\right)=0
$$

where the direction $P_{\mu}$ guarantees an irreducible representation of the Poincare' group for the bilocal field $\mathcal{M}$ [20]. An equation of this type has been used in other approaches to bilocal field dynamics (see ref [20] for other references), but this 'gauge' interpretation of the subsidiary condition [20] provides a more transparent view of the same condition which we have abbreviated as $M Y T P$ above. 
Eq.(A.1) amounts to an effective 3D support to the interaction between the constituents of the bilocal field, which may be alternatively postulated directly for the pairwise BSE kernel $K$ by demanding that it be a function of only $\hat{q}_{\mu}=q-q \cdot P P_{\mu} / P^{2}$, which implies that $\hat{q} . P \equiv 0$. This approach in which the propagators retain their full $4 \mathrm{D}$ forms, allows the use of both the 3D and 4D BSE forms in an interchangeable manner, as shown below.

\section{A.1 3D-4D Interconnection: Spinless Particles}

To demonstrate the basic 3D-4D interconnection under MYTP [12], consider a system of two identical spinless particles, with the BSE [11]

$$
i(2 \pi)^{4} \Phi(q, P)=\left(\Delta_{1} \Delta_{2}\right)^{-1} \int d^{3} \hat{q}^{\prime} M d \sigma^{\prime} K\left(\hat{q}, \hat{q}^{\prime}\right) \Phi\left(q^{\prime}, P\right) ;\left[\Delta_{1,2}=m_{q}^{2}+p_{1,2}^{2}\right]
$$

where the 3D support to the kernel $K$ is implied in its 'hatted' structure:

$$
\hat{q}_{\mu}=q_{\mu}-\sigma P_{\mu} ; \sigma=q \cdot P / P^{2} ; \hat{q} \cdot P \equiv 0 .
$$

The relative and total 4-momenta are related by

$$
p_{1}+p_{2}=P=p_{1}^{\prime}+p_{2}^{\prime} ; 2 q=p_{1}-p_{2} ; 2 q^{\prime}=p_{i}^{\prime}-p_{2}^{\prime} .
$$

The 3D wave function $\phi(\hat{q})$ is defined by [12]

$$
\phi(\hat{q})=\int M d \sigma \Phi(q, P)
$$

When (A.4) is substituted on the RHS of (A.2) one gets

$$
i(2 \pi)^{4} \Phi(q, P)=\left(\Delta_{1} \Delta_{2}\right)^{-1} \int d^{3} \hat{q}^{\prime} K\left(\hat{q}, \hat{q}^{\prime}\right) \phi\left(\hat{q}^{\prime}\right)
$$

Now integrate both sides of this equation wrt $\sigma$ to get an explicit 3D equation

$$
(2 \pi)^{3} D(\hat{q}) \phi(\hat{q})=\int d^{3} \hat{q}^{\prime} K\left(\hat{q}, \hat{q}^{\prime}\right) \phi\left(\hat{q}^{\prime}\right)
$$

where the $3 \mathrm{D}$ denominator function is given by

$$
2 i \pi D^{-1}(\hat{q})=\int M d \sigma\left(\Delta_{1} \Delta_{2}\right)^{-1}
$$

A comparison of (A.5) with (A.6) via (A.7) gives the 3D-4D interconnection

$$
2 i \pi \Delta_{1} \Delta_{2} \Phi(q, P)=D(\hat{q}) \phi(\hat{q})
$$

which directly identifies the RHS as the hadron-quark Vertex Function

$$
\Gamma=D \times \phi / 2 i \pi
$$




\section{Appendix B Salpeter Equation: 3D-4D Interlinkage}

In this Appendix we sketch the main steps [15] to demonstrate the 3D-4D interlinkage of the BS amplitudes which stem from the Salpeter equation [1] for the relativistic hydrogen atom problem, in the notation of the original paper [1] :

$$
i \pi^{2} F\left(q_{\mu}\right) \psi(q)=\alpha \int d^{4} k \mathbf{k}^{-2} \psi(q+k)
$$

A comparison of this equation with eq.(A.6) shows a precise correspondence, except for certain technicalities arising from its fermionic content.Indeed, the 3D nature of the kernel in (B.1) is seen from its dependence on the 3 -vector $\mathbf{k}$, while the quantity $F\left(q_{\mu}\right)$ plays just the role of the product of the two $4 \mathrm{D}$ propagators $\Delta_{1}$ and $\Delta_{2}$ in (A.2): due to the (non-covariant) instantaneous (adiabatic) assumption [1].

$$
F(q)=\left(\mu_{1} E-H_{1}(\mathbf{q})+\epsilon\right)\left(\mu_{2} E-H_{1}(\mathbf{q})-\epsilon\right)
$$

with the time-like components identified as the $\epsilon$ terms! Next, define the 3D wave function $\phi(\mathbf{q})$ by

$$
\phi(\mathbf{q})=\int d \epsilon \psi(\mathbf{q}, \epsilon)
$$

which is the counterpart of (A.4), and use this result to integrate both sides of (B.1) wrt $\epsilon$, after dividing by $F(q)$, so as to get the 3D Salpeter equation [1]

$$
\left[E-H_{1}(\mathbf{q})-H_{2}(\mathbf{q})\right] \phi_{ \pm \pm}= \pm \Lambda_{ \pm}^{(1)} \Lambda_{ \pm}^{(2)}\left(-2 i \pi \Gamma(\mathbf{q})=(-4 i \alpha) \int d^{3} k \mathbf{k}^{2} \phi(\mathbf{q}+\mathbf{k})\right.
$$

where the \pm components are associated with the energy projection operators $\Lambda$ which however do not involve the time-like $\epsilon$.

The crucial aspect, on the other hand, is the $3 \mathrm{D}-4 \mathrm{D}$ interconnection which is obtained by substituting the second part of eq.(B.4) on the RHS of (B.1), after making use of (B.3):

$$
F(q) \psi(q)=\Gamma(\mathbf{q})
$$

where $\Gamma(\mathbf{q})$ is the $3 \mathrm{D}$ BS vertex function. It is the precise fermionic counterpart of the scalar eq.(A.9), since the $F(q)$ function is the product of the two 4D propagators. The form (B.5) is not formally covariant, but this is a mere technicality which can be remedied by standard methods. The reconstructability of the $4 \mathrm{D}$ vertex function from the Salpeter equation was independently noticed by the Bonn group [24]. The MYTP [12] now provides a formal theoretical basis for all Salpeter-like equations in a two - tier form $[21-24]$.

\section{Appendix C: BS Normalizers $N_{P}$ And $N_{V}$}

In this Appendix we outline a derivation of the BS normalizer $N_{H}$ from its 'classical' definition $[58,59]$

$$
\frac{2 i P_{\mu}}{(2 \pi)^{4}}=\int d^{4} q \operatorname{Tr}\left[\bar{\Psi}(q, P) \partial_{P_{\mu}} S_{F}\left(-p_{2}\right)^{-1} \Psi(q, P) S_{F}\left(-p_{2}\right)^{-1}\right]+(1 \Leftrightarrow 2)
$$

which is of course fully equivalent to (3.15) of text. To illustrate the techniques of both the MYTP scenarios, we consider first the pseudoscalar case under $C I A$, followed by the vector case under $C N P A$. 


\section{C.1 Pseudoscalar Meson Normalizer}

To simplify (C.1), note that

$$
\partial_{P_{\mu}} S_{F}^{-1}\left( \pm p_{1,2}\right)= \pm i \gamma_{\mu} \hat{m}_{1,2}+i \gamma_{\mu} \sigma
$$

where $\sigma \equiv q . P / P^{2}$ is the longitudinal fraction of $q$ in the $P$-direction. Substituting in (C.1) from eq.(3.14) of text, and taking the traces, the result for pseudoscalar mesons is

$$
N_{P}^{-2}=2 \int \frac{d^{4} q}{i(2 \pi)^{4}} \frac{D^{2} \phi^{2}}{\Delta_{1}^{2} \Delta_{2}}\left[\left(M^{2}-\delta m^{2}+\Delta_{2}\right)\left(\hat{m}_{1}+\sigma\right)^{2}+\Delta_{1}\left(\hat{m}_{1}+\sigma\right)\right]+(1 \Leftrightarrow 2)
$$

where $\delta m=m_{1}-m_{2}$. Unforfunately the $\left(\hat{m}_{1}+\sigma\right)^{2}$ term in the numerator causes a negative contribution to the $\sigma$-integration. To overcome this problem, one may consider, following Nishijima [60], that the 'charge' is concentrated on one of the quark lines, say $p_{1}$, which amounts to taking the derivative w.r.t. $p_{1}$ instead of w.r.t. $P$ as above. A more symmetrical possibility consists in interchanging $p_{1}$ with $p_{2}$, and weighting these two contributions with the momentum fractions $\hat{m}_{1,2}$ respectively. The result for the pseudoscalar case, after simplification is

$$
\begin{aligned}
N_{P}^{-2}= & 2 \hat{m}_{1} \int \frac{d^{4} q}{i(2 \pi)^{4}} \frac{D^{2} \phi^{2}}{\Delta_{1}^{2} \Delta_{2}}\left[\left(M^{2}-\delta m^{2}+\Delta_{2}\right)\left(\hat{m}_{1}+\sigma\right)\right. \\
& \left.+\Delta_{1}\right]+(1 \Leftrightarrow 2)
\end{aligned}
$$

So far the treatment is quite general. We now specialize to the $M Y T P$ [12] derivation under $C I A$ conditions [11]. Since the normalizer corresponds to an e.m. form factor (with $k_{\mu}=0$ ), it just escapes the problem of time-like components in the gaussian form factors, even in the CIA scenario [33].

The 'pole' integration over $M d \sigma$ may now be carried out as in Sect.2 [23], and the result is a $3 \mathrm{D}$ integral:

$$
N_{P}^{-2}=\int \frac{d^{3} \hat{q} \phi^{2}(\hat{q})}{(2 \pi)^{3}}\left[\left(1-\frac{\delta m^{2}}{M^{2}}\right) G(\hat{q})+\left(1+\frac{\delta m^{2}}{M^{2}}\right) D(\hat{q})\right.
$$

where

$$
G(\hat{q})=2\left(\omega_{1} \omega_{2}+M^{2} \hat{m}_{1} \hat{m}_{2}\right) \omega_{12}
$$

and the other symbols are as defined in Sect.2. The rest of the quadrature is routine, but is skipped for brevity. Since the CNPA techniques are illustrated below (for V-mesons), we give without proof the corresponding Lorentz-invariant result under $C N P A$ [39], viz.,

$$
N_{P}^{-2}=2 M \int \frac{d^{3} \hat{q}}{(2 \pi)^{3}} e^{-\hat{q}^{2} / \beta^{2}}\left[\left(1+\delta m^{2} / M^{2}\right)\left(\hat{q}^{2}-\lambda / 4 M^{2}\right)+2 \hat{m}_{1} \hat{m}_{2}\left(M^{2}-\delta m^{2}\right)\right]
$$

where the internal momentum $\hat{q}=\left(q_{\perp}, M x\right)$ is formally a 3-vector, in conformity with the 'angular condition' $[9,23,35]$. Specializing to equal mass kinematics (needed for $F_{\pi}$ calculation in sect 3.3 ), we have

$$
N_{P}^{-2}=2 M \int_{-1 / 2}^{+1 / 2} M \frac{d x \pi \beta^{2}}{(2 \pi)^{3}} e^{-M^{2} x^{2} / \beta^{2}}\left[\beta^{2}+M^{2} x^{2}+m_{q}^{2}+M^{2} / 4\right]
$$

which works out at

$$
N_{P}^{2}=2 M \frac{\beta^{3}}{(\pi)^{3 / 2}}\left[-\frac{M \beta}{2 \sqrt{\pi}} e^{-M^{2} / 4 \beta^{2}}+\left(3 \beta^{2} / 2+m_{q}^{2}+M^{2} / 4\right) \operatorname{erf}(M / 2 \beta)\right]
$$




\section{C.2 Vector Meson Normalizer}

We next consider the Vector meson normalization directly under the Covariant null plane $(C N P A)$ formalism of Sect.2, and under unequal mass kinematics.

The BS normalizer $N_{V}$ for a V-meson is obtained from (C.1) with the replacement $\gamma_{5} \rightarrow i \gamma . \epsilon$, before evaluating the traces. The $\mathrm{V}$-meson result analogous to (C.2) is

$$
\begin{aligned}
N_{V}^{-2}= & 2 \hat{m}_{1} \int \frac{d^{4} q}{i(2 \pi)^{4}} \frac{D^{2} \phi^{2}}{\Delta_{1}^{2} \Delta_{2}}\left[\Delta_{1}\right. \\
& \left.+\left(M^{2}-\delta m^{2}+2 m_{1}^{2} / 3+2 m_{2}^{2} / 3-2 \Delta_{1} / 3\right)\left(\hat{m}_{1}-\Delta_{1} / 2 M^{2}\right)\right] \\
& +[1 \Leftrightarrow 2]
\end{aligned}
$$

where we have dropped all the $\Delta_{2}$ terms in the first group, anticipating their vanishing on pole integration [23]. We have also averaged over the $\mathrm{V}$-meson polarization according to

$$
<\epsilon_{\mu} \epsilon_{\nu}>=\frac{1}{3} \theta_{\mu \nu} ; \theta_{\mu \nu} \equiv \delta_{\mu \nu}+\frac{P_{\mu} P_{\nu}}{M^{2}}
$$

The last term on the RHS of (C.6) has been simplified from the term

$$
4 \epsilon . p_{1} \epsilon . p_{2} p_{1 \mu}-2 \Delta_{1} \epsilon \cdot p_{2} \epsilon_{\mu}
$$

in the trace calculation, in the same relative normalization as the others (after extracting an overall factor $P_{\mu}$ ), and is explained as follows. Since the only component that survives after integration is in the direction of $P_{\mu}$, the second term (proportional to $\epsilon_{\mu}$ ) vanishes due to $\epsilon \cdot P=0$. For the same reason, we have symmetrically

$$
4 p_{1} . \epsilon p_{2} . \epsilon=-2\left(p_{1} . \epsilon\right)^{2}-2\left(p_{1} . \epsilon\right)^{2}
$$

which on averaging over the vector polarization gives

$$
+2\left(m_{1}^{2}+m_{2}^{2}\right) / 3-2\left(\Delta_{1}+\Delta_{2}\right) / 3-\left[2\left(p_{1} \cdot P\right)^{2}+2\left(p_{2} \cdot P\right)^{2}\right] / 3 M^{2}
$$

In the last term which has a second order of virtuality (in powers of $\Delta_{i}$ ), we invoke the $C N P A$, viz., $p_{-}=-p_{+} M^{2} / P_{+}^{2}$ for each of $p_{1,2}$, so that

$$
\left(p_{i} . P\right)^{2}=\left[\left(p_{i-} P_{+}+p_{i+} P_{-}\right) / 2\right]^{2}=0 ; \quad P_{-}=M^{2} / P_{+} ;
$$

[However we do not take this liberty in the linear terms in $p_{i} . P$ which have only a first order of virtuality]. Thus we take $p_{1 \mu}=-p_{1} \cdot P P_{\mu} / M^{2}$ with

$$
p_{1} \cdot P=\left(\Delta_{1}-\Delta_{2}\right) / 2-M^{2} \hat{m}_{1} ; \quad 2 M^{2} \hat{m}_{1}=M^{2}+m_{1}^{2}-m_{2}^{2}
$$

For integration over $d^{4} q$, it is convenient to convert to new variables as follows [37, 23]

$$
d^{4} q=d^{2} q_{\perp} d p_{2+} d p_{2-} / 2
$$

where the pole integration is first carried out over $p_{2-}$ to give:

$$
\int d p_{2-} \frac{D^{2}}{4 i \pi \Delta_{1}^{2} \Delta_{2}}=2 p_{2+} M^{2} / P_{+}^{2}
$$




$$
\begin{gathered}
\int d p_{2-} \frac{D}{4 i \pi \Delta_{1} \Delta_{2}}=M / P_{+} \\
N_{V}^{-2}=2 \hat{m}_{1} \int \frac{d^{2} q_{\perp} d p_{2+} \phi^{2} M / P_{+}}{(2 \pi)^{3}}\left[D+\left(M^{2}-\delta m^{2}+2 m_{1}^{2} / 3+2 m_{2}^{2} / 3\right) \times\right. \\
\left.\left(2 x_{2} M \hat{m}_{1}-D / 2 M^{2}\right)-2 / 3 \hat{m}_{1} D\right]+[1 \Leftrightarrow 2]
\end{gathered}
$$

where $x_{2}=p_{2+} / P_{+}$and has a range $0 \leq x_{2} \leq+1$, to ensure that the above expression reflects the 'correct' relative positions of the various poles in the $p_{2-}$ plane. The further integrals may be evaluated using gaussian functions. The ground state $L=0$ function $\phi_{0}$ has the gaussian form

$$
\phi(\hat{q})=\exp \left[-\frac{1}{2} \hat{q}^{2} \beta^{-2}\right]
$$

where $\hat{q}^{2}=q_{\perp}^{2}+M^{2} x^{2} ; x=\hat{m}_{2}-x_{2}$ and

$$
D=2 M\left[m_{2}^{2}+q_{\perp}^{2}+M^{2} x^{2}-\hat{m}_{2}^{2} M^{2}\right]
$$

The integration over $d^{2} q_{\perp}$ in (C.6) yields

$$
\begin{aligned}
N_{V}^{-2}= & 2 \hat{m}_{1} \pi \beta^{2} \int \frac{d x_{2} M}{(2 \pi)^{3}} \exp \left[-M^{2} x^{2} / \beta^{2}\right]\left[D_{\beta}+\left(M^{2}-\delta m^{2}+2 m_{1}^{2} / 3+2 m_{2}^{2} / \beta \varnothing \cdot 10\right)\right. \\
& \left.\times\left(2 x_{2} M \hat{m}_{1}-D_{\beta} / 2 M^{2}\right)-2 / 3 \hat{m}_{1} D_{\beta}\right]+[1 \Leftrightarrow 2]
\end{aligned}
$$

where $D_{\beta}$ is obtained from (C.9) by replacing $q_{\perp}^{2}$ with $\beta^{2}$. The final integration over $x_{2}$ is generally an error function after changing the variable from $x_{2}$ to $x=\hat{m}_{2}-x_{2}$, which gives the limits of $x$ integration as

$$
-\hat{m}_{1} \leq x \leq+\hat{m}_{2}
$$

In the simple case of equal mass kinematics, the final result for the normalization is

$$
N_{V}^{-2}(2 \pi)^{3}=2\left(\pi \beta^{2}\right)^{3 / 2} \operatorname{erf}(M / 2 \beta)\left[2 D_{\beta^{*}} / 3+\left(M^{2}+4 m_{q}^{2} / 3\right)\left(M / 2-D_{\beta^{*}} / 2 M^{2}\right)\right]
$$

where

$$
D_{\beta^{*}}=2 M\left[m_{q}^{2}+3 \beta^{2} / 2-M^{2} / 4-\frac{\sqrt{M^{2} \beta^{2} / \pi} \exp \left(-M^{2} / 4 \beta^{2}\right)}{2 \operatorname{erf}(M / 2 \beta)}\right]
$$

For the case of $\rho$, the normalization works out after substituting for the input parameters from eqs (3.4-5) of text, as

$$
N_{\rho}=\frac{4.340 G e V^{-3}}{(2 \pi)^{3 / 2}}
$$

\section{Appendix D: Electroweak Consts $f_{P}$ And $g_{V}$}

In this Appendix we outline, mainly for illustration, short derivations of the pseudoscalar decay constant $f_{P}$ and the $\mathrm{V}$-meson e.m. decay constant $g_{V}$, under the respective premises of $C I A$ and $C N P A$. 


\section{D1 The P-Meson Weak Decay Constant $f_{P}$}

The general formula for $f_{P}$ via 2 -quark loop is given by $[59,23]$

$$
f_{P} P_{\mu}=\sqrt{3} \int d^{4} q \operatorname{Tr}\left[\Psi_{P} \gamma_{\mu} \gamma_{5}\right]
$$

where the factor $\sqrt{3}$ in front represents the effect of color [23]. Substituting from (3.13-14), and taking the traces, the integrand on RHS simplifies to

$$
" T r "=\frac{4}{2 i \pi} D(\hat{q}) \phi(\hat{q}) N_{P}\left[1 ; P_{n} / M\right] \frac{m_{1} p_{2 \mu}+m_{2} p_{1 \mu}}{\Delta_{1} \Delta_{2}}
$$

The next step lies in expressing the 4 -vectors $p_{1,2}$ in the directons parallel and perpendicular to $P_{\mu}$ respectively, and noting that the latter will not survive the $d^{2} q_{\perp}$ integration. The parallel components in turn are

$$
P_{\mu}\left[\hat{m}_{1,2} \pm q \cdot P P^{-2}\right]
$$

The second term is just the longitudinal component $q_{l}=q \cdot P / M$ of $q_{\mu}$ which, in the $C I A$ [11] version of $M Y T P$, directly contributes to the integral in (D.1) via the poles of the propagators $\Delta_{1,2}$ in this variable. In the $C N P A$ version [39] on the other hand, we have

$$
q . P=q \cdot n P_{n}+q_{n} P \cdot n
$$

where the corresponding 'pole' variable is proportional to $q_{n}$ (see text). Then since $d^{4} q=d^{3} \hat{q} q_{n}$, see eq.(2.1), the 'pole' integration over $d q_{l}$ may be carried out exactly as in Appendix A and Sect.2 for $C I A$ and $C N P A$ respectively. Collecting the various factors and simplifying, the result for $f_{P}$, eq.(D.1), may be expressed as a $3 \mathrm{D}$ integral in either case. For definiteness, the $C I A$ result is

$$
\begin{aligned}
f_{P}= & \frac{\sqrt{3} N_{P}}{(2 \pi)^{3 / 2}} \int d^{3} \phi\left[2 m_{12}\left(1-\frac{\delta m^{2}}{M^{2}}\right)\right. \\
& \left.+\frac{\delta m D}{M^{2}}\left(\omega_{2}^{-1}-\omega_{1}^{-1}\right)\right]
\end{aligned}
$$

where $\delta m=m_{1}-m_{2}$ amd $m_{12}=m_{1}+m_{2}$, and the other symbols are as defined in Appendix A. And the 3D integration over $d^{3} \hat{q}$ is a simple gaussian with the necessary substitutions for $\phi$ and $D$-functions from text. The general formula for unequal mass kinematics becomes finally

$$
\begin{aligned}
f_{P}= & \sqrt{3} N_{P} \beta^{3}\left[2 m_{12}\left(1-\frac{\delta m^{2}}{M^{2}}\right)+\frac{2 \delta m}{M^{2}}<M_{\omega}>\right. \\
& \left.\times\left(<\omega_{2}^{-1}>-<\omega_{1}^{-1}>\right)\left(3 \beta^{2}-\frac{\lambda}{4 M^{2}}\right)\right]
\end{aligned}
$$

A very similar result obtains for the $C N P A$ version which we state without proof:

$$
\begin{aligned}
f_{P}= & 2 \sqrt{3} N_{P} \frac{\pi \beta^{2}}{(2 \pi)^{3 / 2}} m_{12}\left(1-\frac{\delta m^{2}}{M^{2}}\right) \\
& \int_{-\hat{m}_{1}}^{+\hat{m}_{2}} M d x \exp \left[-M^{2} x^{2} / 2 \beta^{2}\right]
\end{aligned}
$$


For equal mass kinematics, the formula simplifies to

$$
f_{P}=4 m_{q} N_{P} \beta^{3} \operatorname{erf}\left(\frac{M}{2 \beta \sqrt{2}}\right)
$$

The physics of such quantities is discussed elsewhere [23]. We note in passing that the value of $f_{\pi}$ works out as $112 \mathrm{MeV}$, c.f. $133 \mathrm{MeV}$ [23] for 'half-off-shell' wave function which agrees with experiment [13], except that the latter does not conform to the 'angular condition' $[35,9]$.

\section{D2 The V-Meson E.M. Decay Constant $g_{V}$}

In a similar way, a general formula for the e.m. decay constant of a V-meson via 2-quark loop, is $[59,23]$

$$
\frac{M_{V}^{2}}{g_{V}} \epsilon_{\mu}=\sqrt{3} e_{Q} \int d^{4} q \operatorname{Tr}\left[\Psi_{V} i \gamma_{\mu}\right]
$$

where $e_{Q}$ is the 'charge' of the composite [23, 59]:

$$
e_{Q}^{2}=\frac{1}{2}(\rho) ; \quad \frac{1}{18}(\omega) ; \quad \frac{1}{9}(\phi) ; \quad \frac{4}{9}(J / p s i) ; \quad \frac{1}{9}(v)
$$

and the quark masses are necessarily equal. In this case, the integrand, after taking the traces, becomes

$$
" T r "=\frac{4}{2 i \pi} D(\hat{q}) \phi(\hat{q}) N_{V}\left[1 ; P_{n} / M\right] \epsilon_{\mu} \frac{\left(m_{q}^{2}-p_{1} \cdot p_{2}\right)+2 p_{1} . \epsilon p_{2} . \epsilon}{\Delta_{1} \Delta_{2}}
$$

where we have anticipated that the surviving components of $p_{1,2}$ are in the direction of the polarization vector $\epsilon_{\mu}$ of the $\mathrm{V}$-meson. This time let us evaluate this quantity under the CNPA [39] version of MYTP, following the techniques of Sect.2 of text. We first note the kinematical results

$$
\begin{gathered}
m_{q}^{2}-p_{1} \cdot p_{2}=\frac{1}{2}\left[\Delta_{1}+\Delta_{2}+M^{2}\right] \\
2 p_{1} \cdot \epsilon p_{2} . \epsilon \Rightarrow \frac{2}{3} p_{1} \cdot p_{2} .
\end{gathered}
$$

Next the $\Delta_{1,2}$ terms resulting from these reductions will give zero contributions to the pole integration, since their poles lie on opposite sides of the $q_{n}$-plane;(see Sect.2). Thus the surviving terms in the numerator of "Tr" are independent of $\Delta_{1,2}$. Hence

$$
" T r " \Rightarrow \frac{4}{2 i \pi} D \phi N_{V}\left[P_{n} / M\right] \epsilon_{\mu} \frac{M^{2} / 6+2 m_{q}^{2} / 3}{\Delta_{1} \Delta_{2}}
$$

Recall also the $C N P A$ analogue of the $C I A$ result (A.7) of text:

$$
\frac{1}{2 i \pi} \int d q_{n} \frac{D(\hat{q}}{\Delta_{1} \Delta_{2}}=1
$$

which reduces the RHS of (D.6) to a trivial 3D integral, resulting in

$$
\frac{M_{V}^{2}}{g_{V}}=4 \sqrt{3} N_{V}\left[P_{n} / M_{V}\right]\left(M^{2} / 6+2 m_{q}^{2} / 3\right)\left(2 \pi \beta^{2}\right)^{3 / 2}
$$

For a discussion of the 'physics', see [23]. 


\section{REFERENCES}

[1] (a) E.E.Salpeter, Phys.Rev.87, 328 (1952); (b) M. Levy, Phys.Rev.88, 72 (1952).

[2] A. Logunov and A.N.Tavkhelidze, Nuovo Cimento 29, 380 (1963).

[3] R. Blankenbecler and R. Sugar, Phys.Rev.142, 105 (1966); F.Gross, ibid 1448.

[4] (a) H.Munczek, Phys.Rev.25, 1579 (1982);

(b) Yu.L.Kalinowsky et al, Few-Body Syst.10, 87 (1991).

[5] E.g., C.Itzykson and J.-K. Zuber, Quantum Field Theory, McGraw Hill and Sons Inc, New York, 1980.

[6] S. Weinberg, Phys.Rev.150, 1313 (1966)

[7] Schweber, Bethe and de Hoffmann, Mesons And Fields, Row, Peterson and Co Inc, Evanston, 1955.

[8] (a) V. Kadychevsky, Nucl.Phys.B6, 125 (1968);

(b) V.A. Karmanov, Nucl.Phys.B166,378 (1980).

[9] Review: J.Carbonell et al, Phys.Rep.400, 215 (1998).

[10] Yu. L. Kalinowski et al, Phys.Lett.B231, 288 (1989).

[11] A.N. Mitra and S. Bhatnagar, Int.J.Mod.Phys.A7, 121 (1992).

[12] M.A. Markov, Sov.J.Phys.3, 452 (1940);

H. Yukawa, Phys.Rev.77, 219 (1950).

[13] Particle Data Group, Phys.Rev.D54, July 1-Part I (1996).

[14] E.E.Salpeter and H.A.Bethe, Phys.Rev.84, 1232 (1951)

[15] A.N.Mitra, Invited Talk at the XXIII Intl Workshop on Fundamental Problems in Particle Physics and Field Theory, June 2000, Protvino; also hep-ph/0005128.

[16] H.A.Bethe and E.E.Salpeter, Handbuch der Physik, Vol.35, p88, Springer 1967.

[17] Quarkonia, ed W.Buchmueller, North-Holland 1994.

[18] H.D.Politzer, Phys.Rev.Lett.30, 1346 (1973);

D.J.Gross and F.Wilczek, Phys.Rev.D8, 3633 (1973).

[19] Some typical examples are:

J.M.Cornwall et al, Phys.Rev.D10, 2428 (1974);

H.Kleinert, Phys.Lett.26B, 429 (1976);

D.Ebert and V.N.Pervushin, Teor.Mat.Fiz.(USSR)36, 313 (1978);

D.W.McKay et al, Phys.Rev.D37, 195 (1988).

[20] J.Lukierski and M.Oziwicz, Phys.Lett.69B, 339 (1977).

[21] A.N.Mitra and D.S.Kulshreshtha, Phys.Rev.D26, 3126 (1982). 
[22] A.N.Mitra, Zeits.f.Phys.C8, 25 (1981);

A.N.Mitra and I.Santhanam, ibidC8, 33 (1981).

[23] N.N.Singh et al, Phys.Rev.D38, 1454 (1988);

Review: S.Chakrabarty et al, Prog.Part.Nucl.Phys.22, 143-180 (1989).

[24] C.R.Munz et al, Nucl.Phys.A578, 397 (1994);

J.Hesag et al, ibid A578, 418 (1994).

[25] G. t'Hooft, Phys.Rev.D14, 3432 (1976).

[26] A.N.Mitra, Intl J Mod Phys.A14, 4782 (1999).

[27] S.L. Adler and A.C. Davies, Nucl.Phys.B244, 469 (1984);

Review: C.D.Roberts and A.G.Williams, Prog Part Nucl Phys.33, 477 (1994).

[28] Y. Nambu and G. Jona-Lasino, Phys.Rev.122, 345 (1961).

[29] H.D. Politzer, Nucl.Phys.B117, 397 (1976).

[30] A.N. Mitra and B.M. Sodermark, Int.J.Mod.Phys.A9, 915 (1994).

[31] $q \bar{q}$ : A.Mittal et al, Phys.Rev.Lett.57, 290 (1986); K.K. Gupta et al, Phys.Rev.D42, 1604 (1990);

qqq: A. Sharma et al, Phys.Rev.D50, 454 (1994).

[32] (a) S.R.Chaudhury et al, Delhi Univ. Preprint (1991)-Unpublished; (b) I.Santhanam et al, Intl.J.Mod.Phys.E2, 219 (1993).

[33] A.N.Mitra and K.-C.Yang, Phus.Rev.C51, 3404 (1995);

A.N.Mitra, Int J Mod Phys A11, 5245 (1996).

[34] P.A.M. Dirac, Rev.Mod.Phys.21, 392 (1949)

[35] H.Leutwyler and J.Stern, Ann. Phys.(N.Y.) bf 112, 94 (1978).

[36] R.J.Perry, A.Harindranath and K.Wilson, Phys.Rev.Lett.65, 2959 (1990).

[37] J.B.Kogut and D.E.Soper, Phys.Rev.D1, 2901 (1970);

S.J.Brodsky et al,Phys.Rev.D8, 4574 (1973);

G.t'Hooft,Nucl.Phys.B72, 461 (1974).

[38] P.P.Srivastava, Phys.Lett.448B, 68 (1999).

[39] A.N.Mitra, Phys.Lett.463B, 293 (1999); see hep-ph/9812404 for details.

[40] R. Barbieri and E.Rimiddi, Nucl.Phys.B141, 413 (1978);

G.P.Lepage, SLAC-Preprint no.212 (1978).

[41] G.Brown anD.Ravenhall, Proc.Roy.Soc.A208, 552 (1951)

[42] J.Sucher, Phys.Rev.Lett.55, 1033 (1985);

N.Nimai Singh, Y.K.Mathur and A.N.Mitra, Few-Body Syst.1, 47 (1986). 
[43] A.N.Mitra and I.Santhanam, Few-Body Syst.12, 41 (1992).

[44] A.N.Mitra, PINSA 65A, 527-584 (1999).

[45] A.G.Macfarlane,Rev.Mod.Phys.34, 41 (1981);

J.S.Ball and F.Zachariasen, Phys.Lett.B106, 133 (1981).

[46] M.V.Terentev, Sov.J.Nucl.Phys.15, 665 (1972);

Yu. M. Antipov et al, Phys.Rev.Lett.56, 796 (1986).

[47] A.N.Mitra, N.N.Singh and R.Ramanathan, Pramana,24, 95 (1985).

[48] D.Atkinson and P.Johnson, Phys.Rev.41, 1661 (1990).

[49] J.Gasser and H.Leutwyler, Nucl.Phys.B250, 465, 517, 539 (1985).

[50] V.L.Chernyak and A.R.Zitnitsky, Phys.Rep.112C, 173 (1984).

[51] A.Le Yaouanc et al, Phys.Rev.D29, 1233 (1984).

[52] A.N.Mitra, Intl J Mod Phys.A11, 5245 (1996).

[53] E.g., T. Hatsuda et al, Phys.Rev.Lett.66, 2851 (1991).

[54] H.Pagels and S.Stokar, Phys.Rev.D20, 2947 (1979).

[55] J.Praschifka et al, Intl J Mod Phys.A4, 4929 (1989);

H.J.Munczek and P.Jain, Phys.Rev.D46, 438 (1992);

A.G.Williams et al, Ann.Phys.(NY) 210, 464 (1991);

K.I.Aoki, et al, Phys.Lett.266B, 467 (1991).

[56] A.V.Radyushkin, Phys.Lett.231B, 218 (1991).

[57] G.Preparata, Phys.Rev.D7, 2963 (1973);

H.Pagels, Phys.Rev.D14, 2747 (1976);

J.S.Ball and F.Zachariasen, Phys.Lett.106B, 133 (1981);

J.M.Cornwall, Phys.Rev.D25, 1579 (1982).

[58] S.Mandelstam, Proc.Roy.Soc.A233, 248 (1955).

[59] C.H.L.Smith, Ann Phys (NY), 53, 521 (1969).

[60] K.Nishijima and A.H.Singh, Phys.Rev.162, 1740 (1967). 